\title{
Chlamydia trachomatis infection and risk of cervical intraepithelial neoplasia
}

\author{
Matti Lehtinen, ${ }^{1}$ Kevin A Ault, ${ }^{2}$ Erika Lyytikainen, ${ }^{3}$ Joakim Dillner, ${ }^{4,5,6}$ \\ Suzanne M Garland, ${ }^{7,8}$ Daron G Ferris, ${ }^{9,10}$ Laura A Koutsky, ${ }^{11}$ Heather L Sings, ${ }^{12}$ \\ Shuang Lu, ${ }^{12}$ Richard M Haupt, ${ }^{12}$ Jorma Paavonen, ${ }^{13}$ for the FUTURE I and II Study \\ Group
}

${ }^{1}$ School of Health Sciences, University of Tampere, Tampere, Finland ${ }^{2}$ Department of Gynecology and Obstetrics, Emory University School of Medicine, Atlanta Georgia, USA

${ }^{3}$ National Institute for Health \& Welfare and Department of Dermatology, University of Oulu, Oulu, Finland

${ }^{4}$ Department of Laboratory

Medicine, Lund University,

Malmö, Sweden

${ }^{5}$ Department of Laboratory

Medicine, Karolinska Institute,

Stockholm, Sweden

${ }^{6}$ Department of Medical

Epidemiological \& Biostatistics,

Karolinska Institute, Stockholm,

Sweden

${ }^{7}$ Microbiology and Infectious

Diseases Department, Royal

Women's Hospital, Murdoch

Children's Research Institute,

Australia

${ }^{8}$ Department of Obstetrics and Gynaecology, University of

Melbourne, Melbourne, Victoria, Australia

${ }^{9}$ Department of Family

Medicine, Medical College of

Georgia, Augusta, Georgia, USA

${ }^{10}$ Department of Obstetrics and Gynecology, Medical College of Georgia, Augusta, Georgia, USA ${ }^{11}$ Department of Epidemiology, University of Washington, Seattle, Washington, USA

${ }^{12}$ Department of Research, Merck Sharp \& Dohme, Corp., Whitehouse Station, New Jersey, USA

${ }^{13}$ Department of Obstetrics and Gynecology, University of Helsinki, Helsinki, Finland

\section{Correspondence to}

Matti Lehtinen, School of Health

Sciences, University of Tampere, Tampere 33014,

Finland; IImale@uta.fi

Accepted 8 March 2011 Published Online First 6 April 2011

\section{UNLOCKAD}

This paper is freely available online under the BMJ Journals unlocked scheme, see http://sti. bmj.com/site/about/unlocked.xhtml

\begin{abstract}
Objectives High-risk human papillomavirus (hrHPV) is the primary cause of cervical cancer. As Chlamydia trachomatis is also linked to cervical cancer, its role as a potential co-factor in the development of cervical intraepithelial neoplasia (CIN) grade 2 or higher was examined.
\end{abstract}

Methods The placebo arms of two large, multinational, clinical trials of an HPV6/11/16/18 vaccine were combined. A total of 8441 healthy women aged 15-26 years underwent cervicovaginal cytology (Papanicolaou (Pap) testing) sampling and C trachomatis testing at day 1 and every 12 months thereafter for up to 4 years. Protocol-specified guidelines were used to triage participants with Pap abnormalities to colposcopy and definitive therapy. The main outcome measured was CIN.

Results At baseline, 2629 (31.1\%) tested positive for hrHPV DNA and 354 (4.2\%) tested positive for $C$ trachomatis. Among those with HPV16/18 infection $(n=965 ; 11.4 \%)$ or without HPV16/18 infection $(n=7382,87.5 \%)$, the hazard ratios (HRs) associated with development of any CIN grade 2 according to baseline C trachomatis status were 1.82 (95\% Cl: 1.06 to $3.14)$ and $1.74(95 \% \mathrm{Cl} 1.05$ to 2.90$)$, respectively. The results were comparable when only the 12 most common hrHPV infections were considered, but the excess risk disappeared when the outcome was expanded to include CIN grade 3 or worse.

Conclusion Further studies based on larger cohorts with longitudinal follow-up in relation to the $C$ trachomatis acquisition and a thorough evaluation of temporal relationships of infections with hrHPV types, $C$ trachomatis and cervical neoplasia are needed to demonstrate whether and how in some situations $C$ trachomatis sets the stage for cervical carcinogenesis. Trial registration NCT00092521 and NCT00092534.

\section{INTRODUCTION}

Infection with the highly prevalent oncogenic human papillomaviruses (HPVs) (lifetime risk 70-80\%), most notably with HPV types 16 and 18, is the primary cause of cervical cancer. ${ }^{1}$ Past infection with Chlamydia trachomatis has also been linked to the development of cervical cancer, as demonstrated both in prospective seroepidemiological studies and PCR-based studies using archival cytological materials. $^{2-5}$

The pathogenesis of $C$ trachomatis in cervical intraepithelial neoplasia (CIN) remains unknown, but original observations ${ }^{6}$ and independent, largescale, longitudinal epidemiological studies ${ }^{5}{ }_{8}$ suggest that $C$ trachomatis may be involved in cervical carcinogenesis. By inducing cervical metaplasia $C$ trachomatis infection can provide target cells for acquisition of HPV (especially HPV type 18). ${ }^{4}{ }^{6}$ On the other hand, by causing local immunoperturbation it may interfere with immune surveillance of persistent infection with hrHPV types. ${ }^{9}$ These two alternatives are supported by cohort studies that show that $C$ trachomatis infection is a risk factor for both incident hrHPV infection and persistence of hrHPV DNA. ${ }^{10-13}$ However, evidence of $C$ trachomatis infection increasing the risk of the development of CIN, among those with or without hrHPV infection at the baseline, is still missing. We used a joint cohort from two placebo-controlled trials of a quadrivalent HPV6/11/16/18 vaccine to examine the role of $C$ trachomatis infection as an independent co-factor (possibly related to HPV acquisition) or HPV-dependent co-factor (related to HPV persistence) in the development of CIN.

\section{METHODS}

A cohort of 17622 women aged 15-26 years was enrolled in two multinational trials which evaluated the efficacy and safety of a quadrivalent HPV6/11/16/18 virus-like particle vaccine (GARDASIL/SILGARD, Merck Sharp \& Dohme, Whitehouse Station, New Jersey, USA). ${ }^{14}{ }^{15}$ Both studies were approved by the institutional review boards (ethical review committees) at participating centres and informed consent was received from all the participants enrolled. The study designs and results of the primary hypotheses have been described, following the consort guidelines. ${ }^{14}{ }^{15}$ The trials recruited healthy women who, at enrolment (day 1), reported having had $0-4$ sex partners during their lifetime, except in Finland, where age over 17 years was used as an exclusion criterion.

As the study had no screening phase, the trials allowed the enrolment of women who had previously been or were currently infected with any of the HPV types known to infect the anogenital tract. All participants were tested for cervical cytological abnormalities, and $C$ trachomatis at baseline and 12-monthly intervals in the FUTURE I and FUTURE II trials, respectively, for immediate treatment after which they were again eligible for the follow-up. ${ }^{14}{ }^{15}$ In this study, the final cohort 
consisted of 8441 women who were randomised to the placebo arms of the two trials only, and followed approximately 3.7 years on average, with 25 th and 75 th percentiles of 3.5 and 3.9 years, respectively (maximum follow-up 4.9 years). $C$ trachomatis serology was not performed.

Anogenital swabs collected at baseline, and all tissues collected from definitive therapy and excisions (including biopsy specimens) were tested with a PCR-based assay ${ }^{16-18}$ for 14 HPV types, including the four vaccine types (ie, HPV6, 11, 16 and 18) and 10 other hrHPV types (HPV31, 33, 35, 39, 45, 51, 52, 56,58 and 59) which are the most common hrHPV types in cervical cancer worldwide. ${ }^{19}$ All tissue specimens, including those from definitive therapy and excision (including all biopsy specimens), were subjected to histopathological review by a blinded pathology panel.

This post hoc analysis was performed to assess the independent role of baseline $C$ trachomatis in the development of CIN by comparing results from the HPV-stratified and HPV-adjusted analyses. The following CIN endpoints were evaluated: 1) CIN grade 2 (CIN2) due to any HPV type; 2) CIN2 related to a hrHPV type; 3) CIN grade 3 (CIN3) or adenocarcinoma in situ (AIS) due to any HPV type and 4) CIN3 or AIS related to a hrHPV type. High-risk types included the following $12 \mathrm{HPV}$ types: HPV 16, 18, 31, 33, 35, 39, 45, 51, 52, 56, 58 and 59. In our longitudinal follow-up study, risks of developing CIN2 and CIN3 or AIS were evaluated separately to distinguish early and later associations of $C$ trachomatis with cervical intraepithelial neoplasia. Rates of these endpoints were summarised based on the number of women with a lesion per 100 person-years at risk.

Univariate and multivariate Cox models ${ }^{20}$ were used to analyse the impact of baseline $C$ trachomatis status on the end points. The univariate analysis stratified by HPV baseline status was first used for the evaluation of the impact of baseline $C$ trachomatis status (positive vs negative) on the RR of developing CIN2 and CIN3 or AIS separately in baseline HPV-positive or HPV-negative individuals. Thereafter, the multivariate models adjusting for covariates such as baseline status (HPV positive or negative), age at study entry, number of lifetime sexual partners and smoking status (current smoker, former smoker, never smoked) were used to confirm the nature (HPV dependent or independent) of the $C$ trachomatis associated risk. In the models pertaining to CIN endpoints due to any HPV type, HPV baseline status was defined based on positivity to HPV 16 or HPV 18. In the models of CIN endpoints, due to hrHPV types, HPV baseline status was based on positivity for any of the 12 tested types.

HRs and 95\% CIs were calculated from both the univariate and multivariate models. In the latter the interaction between $C$ trachomatis and baseline HPV status was assessed by including an interaction term in the Cox multivariate model. From this model, the impact of $C$ trachomatis was estimated within each baseline HPV status (HPV negative and HPV positive) group to compare, at the baseline, the HPV-independent and HPV-dependent risk estimates.

\section{RESULTS}

Of the 8812 women randomised to the placebo arms, 8441 had non-missing data for $C$ trachomatis infection at enrolment, with 354 of 8441 (4.2\%) positive for $C$ trachomatis, and 2629 (31.1\%) positive for hrHPV DNA at baseline. The age distribution, age at sexual debut and lifetime number of sex partners were similar between the $C$ trachomatis-positive and $C$ trachomatis-negative women (table 1). Smoking was more common among the former $(32.8 \%$ vs $26.8 \%)$. The frequency of any squamous intraepithelial lesions, HPV16/18 infection and infections with $\geq 1$ of 14 tested HPV types was twofold higher among baseline C trachomatis-positive women compared with $C$ trachomatis negative women (table 1 ).

Crude (univariate analysis) HRs suggested that women with $C$ trachomatis at baseline were about two times more likely to develop CIN2 than those without (table 2). After adjusting (multivariate analysis) for HPV16/18 status, age at study entry, number of lifetime sexual partners and smoking status, women with $C$ trachomatis at baseline were 1.78 times more likely to develop CIN2 due to any HPV type ( $p=0.002$, table 2$)$ than those without $C$ trachomatis. Finally, in the multivariate analysis among women with HPV16/18 infection at baseline, those with $C$ trachomatis at baseline were 1.82 times more likely to develop CIN2 due to any HPV type compared with those without $(\mathrm{p}=0.030$, table 2). A similar point estimate (HR 1.74, $\mathrm{p}=0.033$, table 2) was observed among those without HPV16/18 infection. In corresponding, multivariate analyses among women with at least one of the 12 tested hrHPV types at baseline, those with $C$ trachomatis were 1.59 times more likely to develop CIN2 related to one of the 12 tested hrHPVs compared with those without ( $p=0.033$, table 2$)$, among baseline hrHPV negative women, the point estimate even if not statistically significant was not materially different (HR 1.35).

When the end points were any HPV- or hrHPV-positive CIN3 or AIS, the point estimates in the total univariate analysis were statistically significant (HR for those with vs those without C trachomatis $=1.74,95 \% \mathrm{CI}: 1.10$ to $2.74, \mathrm{p}=0.017,1.82,95 \% \mathrm{CI}$ : 1.15 to $2.87, p=0.010$, respectively, table 3$)$. However, in the multivariate analysis among HPV16/18, the positives also approached unity (HR for those with vs those without C trachomatis $=1.19,95 \% \mathrm{CI}: 0.62$ to $2.28, \mathrm{p}=0.601$, table 3 ). This was true also for the $12 \mathrm{hrHPV}$ associated point estimates (HR for those with vs those without $C$ trachomatis $=1.05,95 \%$ CI: 0.63 to $1.75, p=0.851$, table 3 ).

\section{DISCUSSION}

Our results suggest that if infection with $C$ trachomatis plays an independent co-factor role in the development of cervical neoplasia, ${ }^{21} 22$ the effect is likely to take place at an early stage of cervical carcinogenesis and/or restricted to some cases only. Our data are well in line with previous reports. Studies showing an effect of $C$ trachomatis on late outcomes, such as cervical cancer, have either used long follow-up times or the highest point

Table 1 Baseline characteristics of the study population

\begin{tabular}{ll}
$\begin{array}{l}\text { Chlamydia } \\
\text { trachomatis positive } \\
\text { at day } \mathbf{1}(\mathbf{N}=354)\end{array}$ & $\begin{array}{l}\text { C trachomatis } \\
\text { negative at day } \\
\mathbf{1}(\mathbf{N}=8087)\end{array}$ \\
\hline $20.2 \pm 2.0$ & $20.0 \pm 2.1$ \\
$16.5 \pm 1.8$ & $16.7 \pm 1.9$ \\
$2(2-3)$ & $2(1-3)$ \\
$116 / 354(32.8 \%)$ & $2166 / 8087(26.8 \%)$ \\
$69 / 337(20.5 \%)$ & $860 / 7854(10.9 \%)$ \\
$208 / 354(58.8 \%)$ & $2540 / 8087(31.4 \%)$ \\
$204 / 354(57.6 \%)$ & $2425 / 8087(30.0 \%)$ \\
$81 / 354(22.9 \%)$ & $884 / 8087(10.9 \%)$ \\
& \\
\hline
\end{tabular}
and/or 18

*Denominator includes only those women with a satisfactory day 1 Pap test result. tIncludes HPV6, 11, 16, 18, 31, 33, 35, 39, 45, 51, 52, 56, 58 and 59 . łncludes HPV16, 18, 31, 33, 35, 39, 45, 51, 52, 56, 58 and 59. hrHPV, high-risk HPV. 
Table 2 Multivariate and univariate analysis for risk (HR) of developing cervical intraepithelial neoplasia grade 2 (CIN2) according to baseline Chlamydia trachomatis and high-risk human papillomavirus (hrHPV) DNA status

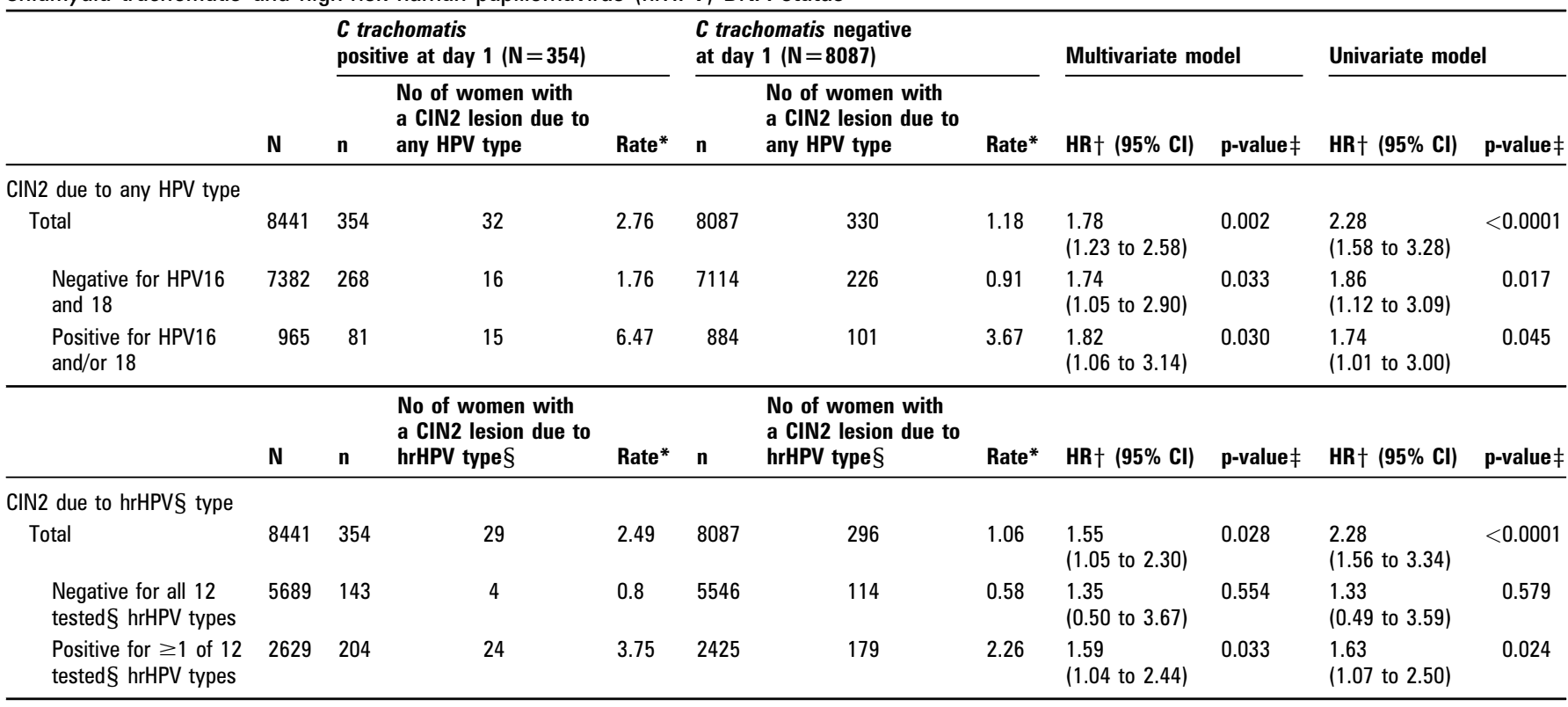

$\mathrm{N}$ : Total number of women with non-missing Chlamydia status and respective PCR status and at least one follow-up visit.

$\mathrm{n}$ : Number of women with positive/negative Chlamydia status and positive/negative PCR status and at least one follow-up visit.

*Number of women with a lesion per 100 person-years at risk.

HHR associated with being positive versus negative to Chlamydia at baseline for each model. For the 'univariate-total' estimates, HR is calculated using the full data. For the 'univariate - negative/positive' estimates, HR is calculated using data from each stratified HPV baseline status group. For the 'multivariate- total' estimates, HR is calculated after adjusting for HPV baseline status (positive or negative), age at study entry, number of lifetime sexual partners and smoking status (current smoker, former smoker, never smoked). Interactions between baseline HPV status and Chlamydia status were tested and proved to be insignificant ( $p$-values of interactions are 0.905 and 0.768 in the models of CIN2 due to any HPV type and CIN2 due to any hrHPV type, respectively). For the 'multivariate-negative/positive' estimates, HR is estimated within each HPV baseline status group after including the interaction term between baseline Chlamydia status and baseline HPV status and adjusting for age at study entry, number of lifetime sexual partners and smoking status.

$\ddagger p$ Value shows how significantly the estimated HRs differ from 1.

§lncludes HPV16, 18, 31, 33, 35, 39, 45, 51, 52, 56, 58, and 59 .

estimates have been obtained for cases with longest lag time between $C$ trachomatis exposure and the outcome. ${ }^{2-5}$

The study has several strengths. This large cohort study enrolled a diverse population of young women participating in developed and developing countries. High diagnostic accuracy for CIN determination was provided by a panel of expert pathologists. In addition, there was well controlled, sensitive testing for HPV DNA and for baseline $C$ trachomatis infection by PCR. Though this was a relatively large study, the quadrivalent vaccine clinical trials were neither designed nor powered to examine the role of $C$ trachomatis infection as a co-factor in the development of CIN. Other limitations of this post hoc analysis study include the limited follow-up time, and the fact that residual confounding by unknown factors could not be eliminated. Overall, any effects of $C$ trachomatis were expected to be seen on the earliest CIN endpoints with the most statistical power.

The questions of which of the two infections, HPV or $C$ trachomatis, has to occur first, and whether the latter increases the risk of acquiring HPV could not be fully answered with the obtained results. The fact that the $C$ trachomatis associated relative risk (RR) of developing CIN2 was statistically significant albeit comparable both before and after acquisition of HPV16/18 (independently of baseline HPV positivity) suggests that the order of the two infections is not important in cervical carcinogenesis. Because of this result (independent risk factor role of $C$ trachomatis) and due to the study setting (screening and treatment of $C$ trachomatis infection during the active follow-up), it is not really possible to make even indirect inferences on the possible increased acquisition of HPV infection following $C$ trachomatis infection. If $C$ trachomatis had been proved to be a risk factor only in baseline HPV negatives or in baseline HPV positives, the increased acquisition hypothesis could have been verified or falsified.
Several cohort studies have indicated that $C$ trachomatis exposure increases the tendency for HPV infection to persist. ${ }^{10-13}$ This effect on persistence could translate to an associated increased risk for cervical cancer. An analysis of the HPV-stratified and HPV-adjusted risk estimates obtained both in the univariate and multivariate analyses (with very limited residual confounding from hrHPVs because of the highly sensitive HPV PCR) suggests that the $C$ trachomatis associated RR of developing CIN2 is comparable both before and after acquisition of HPV. Furthermore, no interaction between the two microorganisms was observed. Thus, $C$ trachomatis may not act in the cervical carcinogenesis by promotion of persistent HPV infection.

In the present study, the observation that baseline $C$ trachomatis positivity was associated with CIN2 but not with CIN3 suggests that $C$ trachomatis infection might facilitate the development of early cervical lesions although there is a possibility that the difference in results for CIN2 and CIN3 might be due to chance. On the other hand, a proportion of both CIN2 and CIN3 lesions regress. This, and the fact that $C$ trachomatis in other longitudinal studies has been associated with early stages of lesions developing to cervical cancer ${ }^{2-6}$ suggest that $C$ trachomatis may have an early role in cervical carcinogenesis in a proportion of cases. The pathobiology of its possible role, however, remains open.

We do not know for how long the $C$ trachomatis-positive individuals had been positive before entering the study. Testing and treatment of $C$ trachomatis infection during the active follow-up, as well as the relatively small number of patients, also reduced the power of our study in assessing $C$ trachomatis associated risks, especially the risk of CIN3 and AIS, longitudinally. The observed CIN2 association could be spurious and due to 
Table 3 Multivariate and univariate analysis for risk (HR, HR) of developing cervical intraepithelial neoplasia grade 3 (CIN3) or adenocarcinoma in situ (AIS) according to baseline Chlamydia trachomatis and high-risk human papillomavirus (hrHPV) DNA status

\begin{tabular}{|c|c|c|c|c|c|c|c|c|c|c|c|}
\hline & \multirow[b]{2}{*}{$\mathbf{N}$} & \multicolumn{3}{|c|}{$\begin{array}{l}\text { C trachomatis } \\
\text { positive at day } 1(\mathrm{~N}=354)\end{array}$} & \multicolumn{3}{|c|}{$\begin{array}{l}\text { C trachomatis negative } \\
\text { at day } 1(\mathrm{~N}=8087)\end{array}$} & \multicolumn{2}{|c|}{ Multivariate model } & \multicolumn{2}{|c|}{ Univariate model } \\
\hline & & $\mathbf{n}$ & $\begin{array}{l}\text { No of women with } \\
\text { a CIN3/AIS lesion due } \\
\text { to any HPV type }\end{array}$ & Rate* & $\mathbf{n}$ & $\begin{array}{l}\text { No of women with } \\
\text { a CIN3/AIS lesion due } \\
\text { to any HPV type }\end{array}$ & Rate* & $\mathrm{HR} \dagger(95 \% \mathrm{Cl})$ & p-value $\neq$ & $\mathrm{HR} \dagger(95 \% \mathrm{CI})$ & p-value $\neq$ \\
\hline \multicolumn{12}{|l|}{ CIN3/AIS due to any HPV type } \\
\hline Total & 8441 & 354 & 20 & 1.71 & 8087 & 267 & 0.96 & $\begin{array}{l}1.31 \\
(0.83 \text { to } 2.06)\end{array}$ & 0.253 & $\begin{array}{l}1.74 \\
(1.10 \text { to } 2.74)\end{array}$ & 0.017 \\
\hline $\begin{array}{l}\text { Negative for HPV16 } \\
\text { and } 18\end{array}$ & 7382 & 268 & 10 & 1.09 & 7114 & 163 & 0.66 & $\begin{array}{l}1.44 \\
\text { (0.76 to } 2.73 \text { ) }\end{array}$ & 0.265 & $\begin{array}{l}1.61 \\
(0.85 \text { to } 3.05)\end{array}$ & 0.146 \\
\hline \multirow[t]{2}{*}{$\begin{array}{l}\text { Positive for HPV16 } \\
\text { and/or } 18\end{array}$} & 965 & 81 & 10 & 4.3 & 884 & 103 & 3.71 & $\begin{array}{l}1.19 \\
(0.62 \text { to } 2.28)\end{array}$ & 0.601 & $\begin{array}{l}1.15 \\
(0.60 \text { to } 2.21)\end{array}$ & 0.666 \\
\hline & $\mathbf{N}$ & $\mathbf{n}$ & $\begin{array}{l}\text { No of women with } \\
\text { a CIN3/AIS lesion due } \\
\text { to hrHPV type } \S\end{array}$ & Rate* & $\mathbf{n}$ & $\begin{array}{l}\text { No of women with } \\
\text { a CIN3/AIS lesion due } \\
\text { to hrHPV type } \S\end{array}$ & Rate* & $\mathrm{HR} \dagger(95 \% \mathrm{CI})$ & p-value $\neq$ & $\mathrm{HR} \dagger(95 \% \mathrm{CI})$ & p-value $\ddagger$ \\
\hline \multicolumn{12}{|l|}{ CIN3/AIS due to hrHPV§ type } \\
\hline Total & 8441 & 354 & 20 & 1.71 & 8087 & 256 & 0.92 & $\begin{array}{l}1.10 \\
\text { (0.69 to } 1.76)\end{array}$ & 0.693 & $\begin{array}{l}1.82 \\
(1.15 \text { to } 2.87)\end{array}$ & 0.010 \\
\hline $\begin{array}{l}\text { Negative for all } 12 \\
\text { tested§ hrHPV types }\end{array}$ & 5689 & 143 & 3 & 0.6 & 5546 & 77 & 0.39 & $\begin{array}{l}1.43 \\
(0.45 \text { to } 4.55)\end{array}$ & 0.540 & $\begin{array}{l}1.50 \\
(0.47 \text { to } 4.77)\end{array}$ & 0.488 \\
\hline $\begin{array}{l}\text { Positive for } \geq 1 \text { of } 12 \\
\text { tested } \S \text { hrHPV types }\end{array}$ & 2629 & 204 & 16 & 2.48 & 2425 & 179 & 2.25 & $\begin{array}{l}1.05 \\
\text { (0.63 to } 1.75)\end{array}$ & 0.851 & $\begin{array}{l}1.09 \\
(0.65 \text { to } 1.81)\end{array}$ & 0.750 \\
\hline
\end{tabular}

$\mathrm{N}$ : Total number of women with non-missing Chlamydia status and respective PCR status and at least one follow-up visit.

$\mathrm{n}$ : Number of women with positive/negative Chlamydia status and positive/negative PCR status and at least one follow-up visit.

*Number of women with a lesion per 100 person-years at risk.

HHR associated with being positive versus negative to Chlamydia at baseline for each model. For the 'univariate-total' estimates, HR is calculated using the full data. For the 'univariate-negative/positive' estimates, HR is calculated using data from each stratified HPV baseline status data. For the 'multivariate-total' estimates, HR is calculated after adjusting for HPV baseline status (positive or negative), age at study entry, number of lifetime sexual partners, and smoking status (current smoker, former smoker, never smoked). Interactions between baseline HPV status and Chlamydia status were tested and proven to be insignificant (P-values of interactions are 0.683 and 0.628 in the models of CIN3/AIS due to any HPV type and CIN3/AIS due to any hrHPV type, respectively). For the 'multivariate-negative/positive' estimates, HR is estimated within each HPV baseline status group after including the interaction term between baseline Chlamydia status and baseline HPV status and adjusting for age at study entry, number of lifetime sexual partners, and smoking status.

$\ddagger \mathrm{p}$ Value shows how significantly the estimated HRs differ from 1.

§lncludes HPV16, 18, 31, 33, 35, 39, 45, 51, 52, 56, 58 and 59 .

confounding by the relationship between $C$ trachomatis and infection with HPV types that have not been tested for. However, the difference in the oncogenicity between the tested and non-tested hrHPV types, and the low prevalence of the latter, ${ }^{23}$ makes this unlikely.

Further studies based on larger cohorts with longitudinal follow-up in relation to the $C$ trachomatis acquisition and

\section{Key Messages}

- We conducted a longitudinal, 4-year follow-up study on the role of Chlamydia trachomatis as a risk factor for cervical intraepithelial neoplasia (CIN) in a sizeable cohort of 8441 placebo vaccinated 15-26-year-old women.

- Baseline C trachomatis PCR positivity was an independent, albeit moderate (HR 1.8) risk factor for the development of CIN2 with materially indistinguishable point estimates in both baseline HPV16/18 positives and HPV16/18 negatives.

- C trachomatis may be involved only in the early stages of cervical carcinogenesis as no increased risk associated with $C$ trachomatis was found for CIN3. The pathobiology of its possible role, however, remains open.

- Further studies based on larger cohorts with longitudinal follow-up in relation to the $C$ trachomatis acquisition and a thorough evaluation of temporal relationships of infections with hrHPV types, $C$ trachomatis and cervical neoplasia are needed to demonstrate whether and how in some situations $C$ trachomatis sets the stage for cervical carcinogenesis. a thorough evaluation of temporal relationships of infections with hrHPV types, $C$ trachomatis and cervical neoplasia are needed to demonstrate whether in some situations $C$ trachomatis sets the stage for cervical carcinogenesis.

Acknowledgements We thank Margaret James, Carolyn Maass, Kathy Harkins and Mary Anne Rutkowski for statistical and programming support and Karyn Davis for publication support.

Funding This study was funded by Merck Sharp \& Dohme, Whitehouse Station, NJ.

Competing interests ML, KAA, JD, SMG, DGF and LAK have received funding through their institutions to conduct HPV vaccine studies for Merck. KAA has received consultancy and advisory board fees from Merck, and has received funding through his institution to conduct HPV-related research for Roche, Gen Probe and

GlaxoSmithKline. JD has received consultancy fees, lecture fees and research grants from Merck and Sanofi Pasteur MSD. SMG has received advisory board fees and grant support from Commonwealth Serum Laboratories and GlaxoSmithKline, and lecture fees from Merck. DGF has received consultancy fees and funding through his institution to conduct HPV vaccine studies for GlaxoSmithKline, and lecture fees from Merck. JP has received consultancy fees and travel grants from Merck and GlaxoSmithKline. HLS, SL and RMH are employees of Merck and potentially own stock and/or stock options in the company.

\section{Patient consent Obtained.}

Ethics approval This study was conducted with the approval of the institutional review boards (ethical review committees).

Contributions This work was supported by Merck Sharp \& Dohme, Whitehouse Station, NJ. This post-hoc analysis was designed by the sponsor in collaboration with external investigators. ML, KAA, JD, SMG, DGF, LAK and JP enrolled patients. SL performed the statistical analysis. All authors were actively involved in the collection, analysis or interpretation of the data, the revising of the manuscript for intellectual content and approval of the final manuscript. All authors, external and internal, had full access to all of the data (including statistical reports and tables) in the study and can take responsibility for the integrity of the data and the accuracy of the data analysis. In addition, all authors have signed a statement confirming they meet the ICMJE criteria for authorship and verify that they had access to all the relevant study data and 
related analyses and vouch for the completeness and accuracy of the data presented. As corresponding author, ML wrote the manuscript and assumes full responsibility for the overall content and integrity of the manuscript.

Provenance and peer review Not commissioned; externally peer reviewed.

\section{REFERENCES}

1. Walboomers JMM, Jacobs MV, Manos MM, et al. Human papillomavirus is a necessary cause of invasive cervical cancer worldwide. J Pathol 1999;189:12-19.

2. Anttila T, Saikku P, Koskela P, et al. Serotypes of Chlamydia trachomatis and risk for development of cervical squamous cell carcinoma. JAMA 2001:285:47-51.

3. Wallin KL, Wiklund F, Luostarinen $T$, et al. A population-based prospective study of Chlamydia trachomatis infection and cervical carcinoma. Int J Cancer 2002;101:371-4.

4. Quint KD, de Koning MN, Geraets DT, et al. Comprehensive analysis of Human Papillomavirus and Chlamydia trachomatis in in-situ and invasive cervical adenocarcinoma. Gynecol Oncol 2009;114:390-4.

5. Naucler P, Chen H-C, Persson K, et al. Seroprevalence of human papillomavirus and Chlamydia trachomatis and cervical cancer risk: nested case-control study. J Gen Virol 2007:88:814-22.

6. Paavonen J, Purola E. Cytological findings in cervical chlamydial infection. Med Biol 1980;58:174-8.

7. Luostarinen $\mathbf{T}$, Lehtinen $\mathrm{M}$, Bjorge $\mathrm{T}$, et al. Joint effects of different human papillomaviruses and Chlamydia trachomatis infections on risk of squamous cell carcinoma of the cervix uteri. Eur J Cancer 2004;40:1058-65.

8. Arnheim L. Immunological Responses in Genital HPV Infections and Etiology of Cervical Cancer. Stockholm, Sweden: Karolinska Institutet, 2005:1-75. http://diss kib.ki.se/2005/91-7140-266-7/thesis.pdf (accessed 22 0ct 2009).

9. Agrawal T, Vats V, Wallace L. Role of cervical dendritic cell subsets, co-stimulatory molecules, cytokine secretion profile and beta-estradiol in development of sequalae to Chlamydia trachomatis infection. BMC Reproduct Biol Endocrinol 2008:6:46.

10. Silins I, Ryd W, Strand A, et al. Chlamydia trachomatis infection and persistence of human papillomavirus. Int J Cancer 2005;116:110-15.

11. Samoff E, Koumans EH, Markowitz LE, et al. Association of Chlamydia trachomatis with persistence of high-risk types of human papillomavirus in a cohort of female adolescents. Am J Epidemiol 2005;162:668-75
12. Velicer C, Zhu X, Vuocolo S, et al. Prevalence and incidence of HPV genital infection in women. Sex Transm Dis 2009;36:696-703

13. Shew ML, Fortenberry JD, Tu W, et al. Association of condom use, sexual behaviors, and sexually transmitted infections with the duration of genital human papillomavirus infection among adolescent women. Arch Pediatr Adolesc Med 2006;160:151-6.

14. Garland SM, Hernandez-Avila M, Wheeler CM, et al. Quadrivalent vaccine against human papillomavirus to prevent anogenital diseases. $N$ Engl J Med 2007;356:1928-43.

15. The FUTURE II Study Group. Quadrivalent vaccine against human papillomavirus to prevent high-grade cervical lesions. N Engl J Med 2007;356:1915-27.

16. International Patent Numbers WO 2003/019143 A2, WO 2006/116276 A2 and WO 2006/116303 A2. http://www.wipo.int/portal/index.html.en. (accessed 5 May 2010).

17. Villa LL, Costa RLR, Petta CA, et al. Prophylactic quadrivalent human papillomavirus (types 6, 11, 16 and 18) L1 virus-like particle vaccine in young women: a randomised double-blind placebo-controlled multicentre phase II efficacy trial. Lancet Oncol 2005;6:271-8

18. Mao C, Koutsky LA, Ault KA, et al. Efficacy of human papillomavirus-16 vaccine to prevent cervical intraepithelial neoplasia: a randomized controlled trial. Obstet Gynecol 2006;107:18-27

19. Li N, Franscheski S, Howell-Jones R, et al. Human papillomavirus type distribution in 30,848 invasive cervical cancers worldwide: variation by geographical region, histological type and year of publication. Int J Cancer 2011;128:927-35

20. Cox DR. Regression models and life tables. J R Stat Soc Ser B Methodological 1972;34:187-220.

21. Koutsky LA, Holmes KK, Critchlow CW, et al. A cohort study of the risk of cervica intraepithelial neoplasia grade 2 or 3 in relation to papillomavirus infection. N Engl J Med 1992;327:1272-8.

22. Lehtinen M, Dillner J, Knekt $\mathrm{P}$, et al. Serologically diagnosed infection with human papillomavirus type 16 and risk for subsequent development of cervical carcinoma: nested case-control study. Br Med J 1996;312:537-9.

23. Schiffman M, Clifford G, Buonaguro FM. Classification of weakly carcinogenic human papillomavirus types: addressing the limits of epidemiology at the borderline. Infect Agent Cancer 2009:4:8.

\section{Minerva}

A 36 year old married man presented with a generalised itchy rash on his hands, ears, and scalp. He also had a dry cough with breathlessness. Bronchoscopy confirmed Pneumocystis jirovecii pneumonia. An HIV test was positive. His CD4 count was $23 \times 10^{6} / 1$ His wife and other family members had also had itchy rashes for months. We diagnosed crusted (Norwegian) scabies. He was treated with permethrin and ivermectin. In ordinary scabies in immunocompetent patients, only 10-15 mites are found whereas in crusted scabies thousands of mites are found, and it is highly infectious. Crusted scabies usually occurs in people who are immunocompromised.

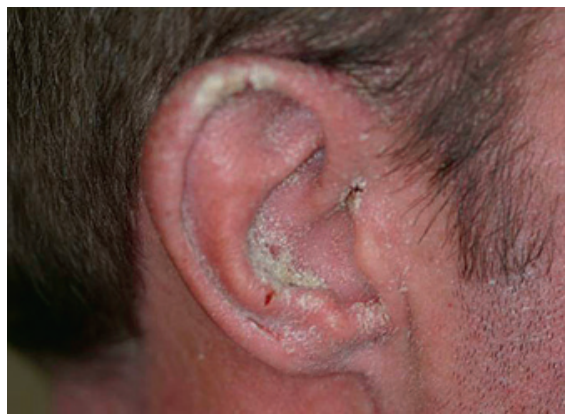

\section{Kathir Yoganathan, Susannah Danino}

Department of Genitourinary and HIV Medicine, Abertawe Bro Morgannwg University Health Board, Singleton Hospital, Swansea SA2 80A, UK

Patient consent Obtained.

Sex Transm Infect 2011:87:376 doi:10.1136/sti.2011.d1906rep 